\title{
LESIONS OF THE CENTRAL NERVOUS SYSTEM IN HERPES ZOSTER
}

\author{
BY
}

\section{IAN R. S. GORDON and JOYCE F. TUCKER}

(From the Royal United Hospital, Bath)

\author{
(RECEIVED 2ND MAY, 1945)
}

SinCE Head and Campbell's classical description of the pathology of herpes zoster, it has been recognized that the principal sites of damage in this disease are the cell bodies of the first sensory neurones in the posterior root ganglia and the ganglia associated with certain of the cranial nerves. More recently, however, it has been emphasized, especially by Lhermitte, that this is not the only lesion possible, and that the virus can attack the central nervous system as well. Herpes zoster, like other virus diseases of the nervous system, may thus be considered as an infection involving the nervous system as a whole, though having a predilection for one particular type of nerve cell. Three cases seen recently seem to illustrate this aspect of herpes zoster and may therefore be of interest.

Cases in which the central nervous system has been affected, normally fall into one of the following groups; firstly, those in which the anterior horns of the spinal cord are affected (anterior poliomyelitis); secondly, those which show evidence of involvement of the pyramidal or other tracts of the white matter of the spinal cord (transverse myelitis); thirdly, those in which the brain is involved (encephalitis). Individual cases may sometimes fall into more than one of these groups.

The three cases seen personally appear to be examples of each of the three groups, the first showing involvement of the lower motor neurones of the second and third lumbar segments following a zoster of the corresponding dermatome; in the second case a paraplegia with sphincter disturbances followed herpes of the 10th and 11th dorsal segments; in the third case a left ophthalmic herpes was followed by a right hemiplegia, aphasia, and pupillary abnormalities.

Case 1.-A.W. Male, aged 68.

History of present illness: He was admitted to hospital complaining of weakness and numbness of the left leg and the left side of the face, which came on in the leg over a period of two to three days about five weeks previously, and seems to have spread from the foot upwards to include the lower part of the abdomen. At the same time as the onset of the weakness and numbness, there appeared a crop of small blisters over the whole of the inner side of the left leg, from below the knee to the groin.
These lasted about a week, leaving a thin papery scar. While the rash was present there was a fairly severe "drawing" pain involving the whole of the left leg. There was no pyrexia, but he slept badly and felt depressed and muzzy in the head.

Two weeks later, he noticed that he could not shut his left eye and that his mouth was crooked. He also complained of a feeling of constriction round his trunk at the level of the iliac crests. He vomited on one occasion but did not complain of headache or disturbance of eyesight or hearing. His appetite was poor and his bowels constipated.

Previous history: Had been abroad for forty years, where he contracted various types of fever. Nothing else significant.

Family history: Nothing significant.

Examination on 22.10 .41 showed a healthy-looking man with normal temperature, pulse rate 82 , regular and of good volume; B.P. 135/95; with no abnormalities in the cardiovascular, respiratory, and digestive systems.

C.N.S.: The fundi were normal; visual acuity and fields normal; pupils equal, regular, reacting to light and accommodation; full eye movements with no nystagmus; slight weakness of movements of the left side of the face; facial sensation and corneal reflexes normal; the other cranial nerves were normal.

In the upper limbs there was no abnormality in muscular power, tone, or coordination, and no tremor. Sensation was normal and the tendon reflexes brisk and equal.

In the trunk, muscular power was normal; touch and pain sensation were slightly diminished over the lower part of the abdomen on both sides. The abdominal reflexes were present on the right but absent on the left side.

In the lower limbs, there was considerable loss of power, most marked in the flexors and adductors of the left hip and in the left quadriceps and hamstrings, less marked in the abductors and rotators of the left hip and the anterior tibials. The glutæi and posterior tibials were apparently unaffected, the tone of the weak muscles was reduced. There was a small area of complete loss of touch, pain and temperature sensation on the inner side of the left knee, and the upper third of the inner side of the shin, with a surrounding area of diminished sensation over the front and inner side of the thigh, and the inner side of the leg down to the internal malleolus on the left side. Vibration and postural sense were unaffected. The left knee jerk was absent, but the left ankle jerk was present and about equal to that on the right side. The plantar reflexes were both flexor.

A lumbar puncture was performed on 25.10.41, and a faintly yellowish fluid was obtained under normal pressure. Other findings were:

Cells: 3 lymphocytes/cu. mm.

Protein: $120 \mathrm{mgms}$. per cent. Globulin positive (Nonne).

W.R.: Negative. 
The W.R. in the blood was also negative.

The abnormal findings were therefore as follows:Slight L. sided facial weakness.

Weakness and hypotonia of both legs.

Area of anæsthesia and analgesia in the L. leg. Absent L. abdominal reflexes.

Absent L. knee jerk.

Xanthochromia and increased protein in the C.S.F.

Progress: During his stay in hospital there was considerable improvement in the weakness and numbness, but he complained of severe neuralgic pains in the left leg necessitating the use of sedatives.

After his discharge home, the weakness of his left leg became much worse again, and he was unable to get about for the next fourteen months. Improvement, however, gradually occurred with massage and active movements, and when seen on 24.6 .43 he was able to walk with the help of crutches. He complained, however, of pins and needles in both hands and of a moderate amount of pain in the left leg. His feet were always cold. Otherwise his health was good.

Examination at this time showed normal fundi; pupils equal and regular, reacting briskly to light and on accommodation; full eye movements with lateral nystagmus; very slight facial asymmetry, but no weakness of the facial muscles was detected; other cranial nerves normal.

No abnormality in power, tone, coordination, sensation or reflexes was found in the upper limbs.

In the trunk there was tenderness on pressure in the 9th intercostal space at the back, but otherwise no abnormality of power or sensation; the abdominal reflexes were all present and equal.

In the lower limbs there was still demonstrable weakness in the left quadriceps, hamstrings, and the rotators of the left hip. The power of the other muscles of the left leg and of the muscles of the right leg was normal. Tone and coordination of all muscles appeared normal. Sensation was normal except for slight diminution of vibration sense in the left leg. The knee and ankle jerks on the left side were both absent even with reinforcement. The plantar reflexes were both flexor. The gait was abnormal; a definite limp was present, though there was no ataxia or Rombergism.

Case 2.- T.P. Male, aged 47. Occupation, fitter.

History of present illness: Fight weeks before admission to hospital he suffered from a typical attack of herpes zoster affecting the skin of the trunk at the level of the 10 th and 11 th dorsal segments.

Four days prior to admission, there was a sudden onset of acute stabbing pain in both loins radiating round to the front of the abdomen. Some hours afterwards his left leg became numb and he was conscious of paræsthesiæ. When he stood up he found he was unable either to walk or stand normally, and his left leg was paralysed. The right leg was weak but not so severely affected. He was also unable to pass his water.

On the day of admission he passed small amounts of urine frequently. The right leg had recovered almost completely and the left leg was recovering some movement.

He had had a transient attack of blurring of vision, but otherwise his previous health had been good with no urinary or dyspeptic symptoms. He denied exposure to syphilis.

Examination on 25.5.42 showed a healthy-looking man with no abnormalities in the respiratory or cardiovascular systems; the bladder could be felt as high as the umbilicus.

C.N.S.: The fundi showed a doubtful temporal pallor of the right disc; there was coarse lateral nystagmus; speech was normal; the other cranial nerves were normal.

In the upper limbs power and tone were normal; tendon reflexes were present and equal; there was slight intention tremor; sensation was normal.

In the trunk the abdominal reflexes were all absent; there were scattered areas of loss of perception to light touch below the umbilicus; pain and deep pressure sense were normal.

In the lower limbs, the right leg showed normal muscular power and tone; the knee and ankle jerks were present; there was no clonus; the right plantar reflex and Oppenheim were extensor; there was no Strumpel and no abnormality of any form of sensation. In the left leg muscular power was almost completely absent; tone was increased with a " cog-wheel " rigidity; the knee and ankle jerks were both exaggerated as compared with the right side; there was no clonus; the plantar reflex and Oppenheim were both extensor, with a positive Strumpel; all forms of sensation were impaired; no muscle wasting was observed in either leg.

A lumbar puncture was carried out and 10 c.c. of clear colourless fluid was obtained under normal pressure. Other findings were:-

Cells: 100 red cells; no white cells.

Protein: 35 mgms. per cent.; Globulin: negative. Lange: 1233400000 .

W.R.: negative.

In brief, the abnormal findings were as follows:-

Doubtful temporal pallor of $\mathbf{R}$. disc.

Nystagmus.

Intention tremor.

Weakness and spasticity of L. leg.

Diminished sensation over abdomen and L. leg. Absent abdominal reflexes.

Increased knee and ankle jerks on $\mathrm{L}$. side.

Plantar reflex and Oppenheim extensor both sides. Retention of urine.

Abnormal Lange curve in C.S.F.

Progress: At first the patient required repeated catheterization. Following discharge from hospital he improved slowly, and finally was able to get about pretty well and walk most of the day without getting tired. There was, however, always some weakness of both legs as compared with his condition previous to admission. The sensation of constriction round the waist persisted and he had had urgency and frequency of micturition. He had only had proper control over his bowels for the last six months. He was now constipated.

For the last six months the weakness and stiffness in the legs had been getting worse again. He got tired easily and could only walk about a mile. The frequency and urgency of micturition had also been worse, as was the pain. His appetite was good and his weight steady. He slept well; there were no flexor spasms or pain at night, though he was disturbed by the frequency. $\mathrm{He}$ had had no further trouble with his eyesight.

Examination on 18.10.44 showed a healthy-looking man; the fundi showed deep physiological cups but were otherwise quite normal; the pupils were moderately dilated, reacted briskly to light and on accommodation; ocular movements full, with no nystagmus; the other cranial nerves were normal.

In the upper limbs, power tone and coordination were normal, reflexes were normal, and there were no sensory abnormalities.

In the trunk the abdominal reflexes were not brisk, but were present and equal; muscular power was normal; there were vaguely defined areas of hypoæsthesia and hypalgesia over the abdomen.

In the lower limbs, there was slight weakness in flexion of the left knee; power was otherwise normal; there was moderate spasticity in both legs; coordination was somewhat impaired. There was gross ataxia and he walked with a wide base; there was slight Rombergism. Sensation was normal apart from some diminution of vibration sense; the tendon jerks were brisk and equal; the plantar reflexes were still both extensor; no Strumpel was present.

Case 3.-H.A. Male, aged 26. Private soldier

History of present illness: About the middle of July 1944 he was admitted to the 30th Gen. Hospital with a left-sided herpes ophthalmicus and œdema of the left eyelid. He complained of general malaise and leftsided frontal headache which he had had for five days. 
On examination at this time there was a herpetic rash over the distribution of the ophthalmic division of the fifth nerve and right submaxillary adenitis but no corneal ulceration. After his evacuation to this country slight ciliary injection developed in the left eye.

Three weeks later he suddenly felt queer and complained of pain in his right arm; there was no unconsciousness but he was unable to raise the arm. A partial paralysis of the right arm and the right side of the face was found on examination; speech was slurred. The paralysis was flaccid but with normal tendon reflexes, no wasting and no sensory abnormalities; it did not involve the right leg or the left side of the body: the plantar reflexes were flexor and the abdominal reflexes present. A lumbar puncture done at this time showed xanthochromia, 3 cells per cu. mm., normal protein and chlorides and a negative W.R.

A week later the paralysis of the right arm became more pronounced and the right leg began to be affected; the pupils became unequal, the left being larger than the right but both reacting to light and on accommodation; speech became gradually more and more difficult, developing into a gross aphasia.

The herpes had almost cleared before the onset of the paralysis.

Previous history and family history yielded nothing significant.

Examination on 3.9.44 showed a well-developed man with no abnormality in heart, lungs, or abdomen. His temperature was 97 , pulse rate 80 , regular and of good volume; B.P. $130 / 84$; urine normal.

A fairly gross degree of aphasia was still present, though it appeared to have improved slightly recently. The defect was predominantly expressive; he could form simple sentences but often got held up for lack of a word; he complained of difficulty in thinking of the right word to say, though he knew what he wanted to say and could recognize the word when it was said for him; he often used wrong words and pronounced them badly; reading was very slow and inaccurate; memory seemed fairly good, though he could not give a connected history; he could repeat words accurately and he could obey commands as long as they are not too complicated, but he had difficulty in distinguishing between left and right and in calculation. He had little nominal defect; he cooperated well and there was no confusion or disorientation. He stated that he had noticed that his speech became worse if he was tired or if he had been trying to read for a long time.

There was bilateral diminution in the sense of smell; a gross refractive error was present in the right eye and a slight one in the left eye; the discs were normal; the visual fields appear normal to rough tests; the pupils were equal and reacted briskly to light but only sluggishly on accommodation; eye movements were full with no ptosis or nystagmus; there was a right-sided upper motor neurone paralysis of the face; facial sensation was normal; the right trapezius was weak and the tongue was protruded to the right.

In the upper limbs, muscular power, tone, and coordination and all forms of sensation were normal in the left arm. There was weakness of all movements of the right arm; he could just flex the wrist, and could abduct the shoulder to a right angle, but the movement was definitely weak; muscle tone was increased; sensation was normal except for defective postural sense; there was no astereognosis present. The tendon reflexes were brisker on the right than on the left.

In the trunk, power and sensation were normal, but the right lower abdominal reflex was diminished.

In the lower limbs there was a just detectable weakness of movements at the right ankle and knee; tone was equal in both legs; there was some tremor in both legs but no clonus; posture and coordination were otherwise normal. There was no abnormality of sensation in either leg; the tendon reflexes were brisker on the right side than on the left; plantar reflexes were both extensor. The gait was of the typical hemiplegic type and there was some degree of pes cavus on both sides, more so on the right than on the left.
The following investigations were carried out:X-ray of skull: normal.

Lumbar punctures:

27.8.44.

Clear fluid; pressure, $140 \mathrm{~mm}$.

Cells: 70 red cells; 12 white cells per cu. mm.

Protein: $60 \mathrm{mgm}$. per cent.

Sugar: $53 \mathrm{mgm}$. per cent.

4.9.44.

Clear fluid; pressure: $60 \mathrm{~mm}$. with rapid rise and fall on jugular compression.

Cells: 2 polymorphs and 36 lymphocytes per cu. $\mathrm{mm}$.

Protein: $70 \mathrm{mg}$. per cent. Globulin positive (Nonne.)

Lange: 1223320000 .

Cisternal encephalography showed both lateral and the 3rd ventricle to be well filled and central in position. No evidence of a space occupying lesion was found.

In brief, the abnormal findings were:-

Aphasia.

Diminished sense of smell.

Pupils reacting sluggishly on accommodation.

R. hemiplegia with spasticity and increased tendon reflexes.

Bilateral extensor plantar reflexes.

Tremor of legs.

Diminution in postural sense in both arms.

Pleocytosis, positive globulin, increased total protein and abnormal Lange curve in the C.S.F.

Progress: The movements of the right elbow and shoulder improved rapidly, and when examined on his discharge from hospital on 21.9.44, there was marked general improvement. The aphasia, however, was still quite well marked, though he could talk sufficiently well to make himself understood. Fine movements of the right hand and fingers were still very poor, though movements at the right shoulder were fairly normal; leg movements were good and he could walk fairly well.

As none of these cases have died it has not been possible to investigate the pathological changes responsible for the clinical findings. It may be of interest, therefore, to review the findings of other authors in similar cases and the evidence they adduce in favour of lesions of the central nervous system in herpes zoster. They will be found to afford considerable support for the theory that the zoster virus can invade the central nervous system and to suggest a possible pathological basis for the clinical findings observed in the three cases just described.

Involvement 'of the anterior horn and the lower motor neurone in herpes is not uncommon. The site of the paralysis is usually segmental and related to that of the eruption. It is of the lower motor neurone type and comes on as a rule a few days after the appearance of the eruption and may persist for a period of years, though the ultimate prognosis according to Bing (1939) is always favourable. Cases of this type are described by Joffroy (1882), Waller (1885), Taylor (1895), Hardy (1876), Cornil (1930), Wilson (1940), and Carter and Dunlop (1941).

Cases showing involvement of the white matter of the spinal cord are rare. A monoplegia with or without sensory changes is the commonest clinical picture, but a complete paraplegia with sphincter disturbances. root pains, and complete sensory loss kelow the level of the rash has been described. 
The symptoms of these cord lesions come on gradually as a rule from two to six weeks after the zoster and thereafter slowly improve, though signs of pyramidal involvement, such as weakness, extensor plantar reflexes, increased tendon reflexes which may be responsible for considerable disability, may persist for several years. The prognosis must therefore be regarded as uncertain.

In two cases of spinal cord symptoms following herpes zoster evidence of independent lesions have been discovered. This suggests that in certain cases the herpes occurs secondarily to an independent cord lesion which has been undetected until the herpes draws attention to it. The condition of "symptomatic herpes" is well known, and the whole question will be discussed more fully later on. The occurrence of this type of case is reviewed by Worster Drought and McMenemey (1934).

Evidence of damage to the brain following herpes zoster is also uncommon. The clinical picture in these cases is of two types, firstly a localized lesion very similar to the lesions in the spinal cord, usually resulting in a hemiplegia, and secondly a generalized encephalitis. Most of the cases of the first type have occurred in old age and have had a uniformly fatal outcome. Such cases are described by Fumery and Brissaud (quoted by Worster Drought and McMenemey 1934), by Lhermitte and Vermes (1930) and by Faure-Beaulieu and Lhermitte (1929).

The second type of case appears to be almost universally fatal. They have been described by Schiff and Brain (1930), Thalhimer (1924), Gundersen (1925), and Biggart and Fisher (1938). Netter (1930) has described cases in which the encephalitis preceded the zoster, but these are probably to be regarded, according to Schiff and Brain, as cases of "symptomatic herpes."

Most of these cases show evidence of meningitis as well, and indeed there is evidence that this occurs at some stage in the majority of cases of zoster, though it may be without clinical signs; at any rate a mild lymphocytosis and increase in protein in the C.S.F. is a common finding (Brain, 1940, Wiegmann, 1934).

As regards the pathological basis for these clinical findings the only changes in the central nervous system described by Head and Campbell (1900) were the degeneration of the posterior columns resulting from the destruction of the cell bodies in the posterior root ganglia, but they admit that herpes zoster is probably an acute specific disease of the nervous system as a whole with an initial febrile stage analogous to that of anterior poliomyelitis.

Lhermitte and his colleagues on the other hand have shown that even in uncomplicated cases, herpes zoster results not only in lesions of the posterior root ganglia, but also causes inflammation of the anterior horns of those segments of the cord in relation to the affected ganglia. Sections of the spinal cord show very well marked dilatation of the vessels, destruction of the nerve cells and fibres, neuroglial proliferation and scattered collections of lymphocytes ; these changes are most marked in the region of the posterior horn of the grey matter, but also affect the base of the anterior horn, which shows loss of cell bodies and scattered lymphocytes. While they are most severe in the segment affected by the zoster, they extend to affect adjacent segments to a lesser degree (Lhermitte and Vermes 1930). Similar changes have been described more recently by Denny Brown, Adams and Fitzgerald (1944). These authors emphasize microglial proliferation with lymphocytic infiltration round the veins, which, though strictly unilateral, extends up and down the cord for two or more segments from that in which the eruption occurs. Damage to the nerve cells of the cord is slight but quite definite, and occurs particularly in the anterior horns, substantia gelatinosa, and Clarke's column. These authors also found, in two cases, degenerative changes in the anterior roots at the point at which they pass closest to the posterior root ganglia. They considered that these were far too extensive to be accounted for by the degenerative changes in the anterior horns and postulate that they are due to direct extension of the inflammatory process from the posterior root ganglion.

As regards damage to the white matter of the cord, Lhermitte and Nicolas (1924) found perivascular "cuffing" in the region of the pyramidal tract. Degenerative changes occur of course in the dorsal columns as a result of the damage to the cells. of the posterior root ganglia.

Lhermitte and Vermes also describe the lesions they found in the brain stem and basal ganglia in cases of herpes zoster. In these the vessels are tortuous and distended with blood, there are scattered hæmorrhages, which, though small are numerous, and areas which appear translucent on section owing to the presence of "lacunæ," particularly in the region of the pyramidal tracts. In spite of the advanced age of most of the patients in which these lesions were found, these authors consider that they are directly due to the herpes virus on the grounds that they are of a similar type to the lesions in the spinal cord, and do not resemble the lesions due to arteriosclerosis or thrombosis.

Biggart and Fisher (1938) describe the pathological findings in their fatal case of encephalitis. following herpes zoster. To the naked eye the only visible change was injection of the meninges. On section, abnormalities were found not only in the brain but in the cord as well. The latter showed a diffuse proliferation of the microglia and an infiltration of lymphocytes in the posterior horns. There was no perivascular demyelinization, but the nerve cells showed chromatolysis. In the anterior horns there was no inflammatory reaction, but the cells showed loss of Nissl's substance. The brain showed perivascular infiltration in the medulla, cerebellar nuclei, thalamus, and hypothalamus. The substantia nigra was not involved and there was no predilection for the periadqueductal grey 
matter. There were numerous small perivascular hæmorrhages (more than in the spinal cord). The cytoplasm of the nerve cells showed hyaline changes and in the cortex a variable amount of chromatolysis was present, though perivascular hæmorrhages were absent. In Thalhimer's case the histological findings were similar and the main feature was infiltration with small round cells throughout the whole central nervous system.

These results show that the conception of herpes zoster as an infection of the nervous system as a whole, rather than as a localized disease of the posterior root ganglia rests on a firm pathological basis. It was put forward tentatively by Head and Campbell, who regarded herpes zoster as analogous to acute anterior poliomyelitis in having an initial febrile phase indicative of a general systemic infection, followed by signs of damage to a specific type of cell, and they suggested that it might be regarded as an acute posterior poliomyelitis. The evidence which has been accumulated since that time allows us to push this analogy further and to suggest that there may be a stage of generalization in which the virus is disseminated throughout the central nervous system. In the majority of cases the signs are only those of damage to the posterior root ganglion for which the virus has a special affinity. In a few cases, on the other hand, there is evidence that damage has been done to the rest of the nervous system, and it is this type of case which is considered in this paper. This view is also supported by the frequent occurrence of signs of meningitis in cases of herpes zoster, either in the form of headache, neck rigidity and a positive Kernig's sign, or a pleocytosis and an increase of protein in the C.S.F. The latter finding might possibly be more frequent than it is, if lumbar punctures were done in more cases of herpes zoster.

The first of the three cases described in the paper is an example of a lower motor neurone lesion due presumably to a spread of inflammation from the posterior root and the posterior horn to the anterior horn of the same segment. The distribution of muscular weakness (mainly lumber 2 and 3 ) corresponds well enough to the area of skin affected by the herpes. As has been seen already, such cases are not uncommon, though those affecting the lumbar segments seem to be the least common type. The cerebrospinal fluid findings were interesting, the slight xanthochromia and protein-cell dissociation being more suggestive of a neoplastic or vascular lesion than an inflammatory one. Such C.S.F. findings do, however, occur in the later stages of acute anterior poliomyelitis, and in this case the lumbar puncture was performed five weeks after the onset of symptoms. In other similar cases a moderate pleocytosis has been described (Carter and Dunlop, 1941). The progress of this case indicates that recovery does ultimately tend to occur, though it may be slow, intermittent, and incomplete, which agrees with the results in similar cases described by others. In this case the existence of some degree of facial weakness on the same side as the weakness of the leg and the herpes, and of symptoms such as insomnia, depression, and "muzziness" are at the least suggestive of a more widespread involvement of the nervous system.

The pathogenesis of these cases would seem to be that of an anterior poliomyelitis directly due to the herpes virus in which there is a considerable degree of recovery possible, probably greater than in acute infective anterior poliomyelitis, but in which some of the anterior horn cells nevertheless suffer irremediable damage. The available evidence suggests, however, that damage to the anterior root or even to the nerve trunk may also account for some of the cases of lower motor neurone paralysis following herpes zoster.

The second case represents an acute transverse lesion of the spinal cord with a paraplegia and loss of sphincter function occurring about seven to eight weeks after herpes zoster of the 10th and 11th dorsal segments. The mode and time of onset make it unlikely that in this case we are dealing with an inflammatory lesion due to spread of the herpes virus from the posterior root ganglion. It is much more likely that in this case there was some separate pathological process occurring either as a result of, or coincidentally to, the herpetic inflammation. In this connection the suggestion of Bruce (1907) that the wide extent of the lesion in many of the cases of spinal lesions following herpes zoster, might be accounted for by an inflammatory thrombosis of the postero-lateral or posterior cornual arteries is interesting. Furthermore, many of the lesions described in the brain stem and basal ganglia by Lhermitte and Vermes were hæmorrhagic and destructive in nature, though these authors emphasize that they were not associated with foci of degeneration and hyperæmia, and considered that they were not due to arterial or venous thrombosis. Finally, vascular dilatation and hæmorrhage are frequent findings in the spinal cord in cases of zoster. There would thus seem to be considerable support for the view that herpes zoster can lead to vascular lesions in the cord and brain, and it is possible to argue that in this case the acute myelitis might have been due to a thrombosis or hæmorrhage.

The fact, however, that there was a considerable degree of recovery followed by a further deterioration raises a further possibility. It is well known that herpes zoster can occur secondarily to some other disease of the central nervous system, such as trauma, neoplasm, or inflammation due to other agents, a condition known as "symptomatic herpes." In a case described by Kamman (1928) an intradural cyst was found on laminectomy, and Alajouanine and Griffith (1931) describe the case of a girl of 17 who developed typical signs of disseminated sclerosis two years after a "herpes myelitis." In our case the onset of the herpes and the paraplegia was preceded by a transient attack of blurring of vision; examination showed in addition to the paraplegia, nystagmus, doubtfu! 
temporal pallor of the discs and intention tremor; in the C.S.F. there was a slight abnormality in the Lange curve with a negative W.R. These signs were not confirmed when he was seen two years later, but the possibility remains that his cord lesions might have been due to Disseminated Sclerosis, as in Alajouanine and Griffith's case. An acute transverse myelitis, while perhaps an uncommon feature of Disseminated Sclerosis, does occur (Wilson, 1940).

Though it is difficult to be certain in this case what the exact pathogenesis is, good evidence exists that zoster can cause lesions in the spinal cord affecting both the grey and the white matter, and which may be termed a zoster myelitis, though whether this condition is directly due to the action of the virus or to the indirect effect of vascular lesions must be left open.

The third case is perhaps the most interesting of the three. Following a herpes of the ophthalmic division of the trigeminal nerve, the patient developed a right hemiplegia with an aphasia three weeks after the appearance of the rash. The occurrence of an aphasia after herpes zoster has not, so far as we have been able to discover, been described before. It was of the expressive type and improved rapidly as did the hemiplegia. The C.S.F. showed a definite pleocytosis and increased protein, and in the examination carried out before admission, a xanthochromia. It is possible to conceive that the herpes zoster and the ensuing hemiplegia and aphasia were entirely unconnected and that their association was merely coincidence. It was unfortunately not possible to carry out complement fixation tests on the C.S.F. as was done by Schiff and Brain (1930), but nevertheless it seems difficult to account for the occurrence of a sudden hemiplegia in an otherwise healthy young adult of 26 , with no evidence of arteriosclerosis elsewhere and no source of embolism. It therefore seems to us a reasonable assumption that the two conditions were associated and that the lesion was likely to be of the type described by Lhermitte and Vermes and by Faure-Beaulieu and Lhermitte (1929) in the brain stem and basal ganglia, rather than of that described by Biggart and Fisher (1938); that is, a localized hæmorrhagic or destructive focus affecting the left pyramidal tract in its course between the cortex and mid-brain. The symptoms and course are quite unlike those of the generalized encephalitis occurring in Biggart and Fisher's or Schiff and Brain's cases, where there was drowsiness, confusion, and disorientation rapidly progressing to coma and death; a type of clinical picture analogous to that seen in epidemic encephalitis, or the encephalitis due to the viruses of the acute exanthemata. The sudden onset, rapid recovery and the xanthochromia of the C.S.F. are in favour of a "vascular" type of lesion. In the cases described by Lhermitte and his colleagues, the picture was considerably complicated by the age of the patients ( 75 and 80 ), which suggest that senile arteriosclerotic changes might be responsible for the lesions, and by the presence of a positive W.R. in the C.S.F. of one of the cases. Although these authors make out a very good case for supposing that the lesions found post-mortem were in fact due to the herpes virus, though they might be predisposed to by the existence of vessels " which were weak, both easily ruptured and easily obliterated," their conclusions that such lesions always occur in old people, and that the state of the vessels is a necessary predisposing factor in their production, might seem to be invalidated by this case, where a lesion which appears to be of a similar type (though it has been impossible to verify this pathologically) occurs in a man of 26 . The advanced age of the other cases of this type which have been described has made it difficult to assess prognosis fairly, but in young people it would not appear from this case to be uniformly bad, either as regards life or recovery of function. The site of the original zoster does not necessarily appear to have any connection with that of the brain lesion, since in both of Lhermitte's cases the herpes was spinal. However, in the cases of generalized encephalitis following zoster, Schiff and Brain point out that there may be meningeal symptoms with a local predominance corresponding to the site of the original zoster. In these cases they stress the possibility that the herpes may be "symptomatic" to an encephalitis of other ætiology, but conclude that cases in which the zoster clearly precedes the encephalitis can be considered as cases of zoster encephalitis. Biggart and Fisher consider that in such cases the virus may invade the brain along preformed nervous pathways, in view of the fact that the lesions in their case were particularly marked in the parietal cortex.

In all these cases of generalized encephalitis the lesions demonstrated at autopsy have in common the occurrence of perivascular hæmorrhages and destruction of the nerve cells. Several authors have commented on the fact that these features are characteristic of the lesions of herpes zoster wherever they occur, in the posterior root ganglia, spinal cord or brain. How far this can be taken to indicate that these lesions are vascular in origin is doubtful. While it seems clear that the herpes virus can give rise directly to inflammatory lesions in the course of a generalized invasion of the central nervous system, consideration of many of the cases described in the literature and perhaps especially cases 2 and 3 of this series, would seem to suggest that herpes zoster may lead to an inflammatory thrombosis or hæmorrhage, which is itself responsible for the sudden onset of a hemiplegia or paraplegia several weeks after the original infection. In support of this are the recent observations of Denny Brown, Adams and Fitzgerald (1944), who describe so complete a necrosis of both parenchymatous and interstitial tissues in the posterior root ganglia of a case of herpes zoster, that the appearance was that of an infarct necrosis. They conclude therefore that vascular occlusion was the cause, especially as they were able to demonstrate a thrombosis of the main vessel to the ganglion. 


\section{Summary}

Three cases of herpes zoster complicated by paralysis and other symptoms indicative of involvement of the central nervous system are described and their pathogenesis discussed.

\section{REFERENCES}

Alajouanine, T., and Griffith, B. (1931). Rev. Neurol., 38, i, 84.

Biggart, J. H., and Fisher, J. A. (1938). Lancet, $2,944$. Bing, R. (1939). Textbook of Nervous Diseases. E. V. Mosby, St. Louis and London.

Brain, W. R.(1940). Ibid., Oxford Medical Publications, London.

Bruce, A. (1907). Rev. Neurol. Psychiat.. 5, 885.

Carter, A. B., and Dunlop, J. B. W. (1941). Brit. med. $J ., 1,234$.

Cornil, L. (1930). Rev. Neurol., 37, i, 280.

Denny-Brown, D., Adams, R. D., and Fitzgerald, P. J. (1944). Arch. Neurol. Psychiat. (Chicago), 51, 216.
Faure-Beaulieu, M., and Lhermitte, J. (1929). Rev. Neurol., 36, i, 1250.

Gunderson, E. (1925). Norsk. Mag. Laegevidensk., 86, 105.

Hardy, M. (1876). Gaz. Hôp., Paris, 49, 827.

Head, H., and Campbell, A. W. (1900). Brain, 23, 353.

Joffrey, A. (1882). Arch. Physiol., 9, 170.

Kamman, G. R. (1928). J. Amer. med. Ass., 91, 320.

Lhermitte, J., and Nicholas, M. (1924). Rev. Neurol., 31, i, 361.

- and Vermes, V. (1930). Ibid., 37, i, 1231.

Netter, A. (1930). Bull. Soc. méd. Hôp., Paris, 46, 793.

Schiff, C. I., and Brain, W. R. (1930). Lancet, 2, 70.

Taylor, F. (1895). Guy's Hosp. Rep., 52, 37.

Thalhimer, W. (1924). Arch. Neurol. Psychiat. (Chicago), 12, 73.

Waller, G. (1885). Brit. med. J., 2: 560.

Wiegmann, F. (1934). Münch. med. Wschr., 81, 1970.

Wilson, S. A. K. (1940). Neurology, Edward Arnold and Co., London.

Worster Drought, C., and McMenemey, W. H. (1934). J. Neurol. Psychopath., 14, 52. 